\title{
1 No part gets left behind: Tiled nanopore sequencing of whole ASFV genomes
}

$4 \quad$ Running Title: Tiled amplicon sequencing with improved assembly of African Swine Fever

5 Virus

$7 \quad$ Amanda Warr ${ }^{\mathrm{a}}$, Caitlin Newman ${ }^{\mathrm{a}}$, Nicky Craig ${ }^{\mathrm{a}}$, Ingrida Vendelè ${ }^{\mathrm{a} \bullet}$, Rizalee Pilare $^{\mathrm{b}}$, Lilet

8 Cariazo Cruz ${ }^{\mathrm{b}}$, Twinkle Galase Barangan ${ }^{\mathrm{b}}$, Reildrin G Morales ${ }^{\mathrm{d}}$, Tanja Opriessnig ${ }^{\mathrm{a}}$, Virginia

9 Mauro Venturina ${ }^{b}$, Milagros R Mananggit ${ }^{c}$, Samantha Lycett ${ }^{a}$, Clarissa YJ Domingo ${ }^{b}$,

10 Christine Tait-Burkard ${ }^{\text {a\#}}$

12 a , The Roslin Institute and Royal (Dick) School of Veterinary Studies, University of

13 Edinburgh, Easter Bush, Midlothian, UK

14 b, College of Veterinary Science and Medicine, Central Luzon State University, Science City of Muñoz, Nueva Ecija, The Philippines

c, Regional Animal Disease Diagnostic Laboratory, Department of Agriculture Regional Field

17 Office III, City of San Fernando, Pampanga, The Philippines

$18{ }^{\mathrm{d}}$, Bureau of Animal Industry, Department of Agriculture, Visayas Avenue, Diliman, Quezon

19 City, The Philippines

•Current address: Thermo Fisher Scientific, Vilnius, Lithuania

\# Address correspondence to Christine Tait-Burkard, christine.burkard@roslin.ed.ac.uk . 


\section{Abstract}

24 African Swine Fever virus (ASFV) is the causative agent of a deadly, panzootic disease, infecting wild and domesticated suid populations. Contained for a long time to the African continent, an outbreak of a particularly infectious variant in Georgia in 2007 initiated the spread of the virus around the globe, severely impacting pork production and local economies. The virus is highly contagious and has a mortality of up to $100 \%$ in domestic pigs. It is critical to track the spread of the virus, detect variants associated with pathology, and implement biosecurity measures in the most effective way to limit its spread. Due to its size and other limitations, the 170-190kbp large DNA virus has not been well sequenced with fewer than 200 genome sequences available in public repositories. Here we present an efficient, low-cost method of sequencing ASFV at scale. The method uses tiled PCR amplification of the virus to achieve greater coverage, multiplexability and accuracy on a portable sequencer than achievable using shotgun sequencing. We also present Lilo, a pipeline for assembling tiled amplicon data from viral or microbial genomes without relying on polishing against a reference, allowing for structural variation and hypervariable region assembly other methods fail on. The resulting ASFV genomes are near complete, lacking only parts of the highly repetitive 3'- and 5'telomeric regions, and have a high level of accuracy. Our results will allow sequencing of ASFV at optimal efficiency and high throughput to monitor and act on the spread of the virus. 
Main

43

44

African Swine Fever (ASF) is a viral hemorrhagic disease leading to an extremely high mortality of up to $100 \%$ within $7-10$ days. Clinical signs of infection are non-specific, including fever, ataxia, anorexia, cyanosis, respiratory symptoms, gastrointestinal symptoms and death ${ }^{1}$. The causative agent of ASF is African Swine Fever Virus (ASFV), the only member of the Asfivirus genus and the Asfarviridae family. The virion is large and complex with a diameter of around 175-215nm containing a large, double stranded DNA genome of around $170-190 \mathrm{~kb}$ encoding over 150 open reading frames ${ }^{1,2}$.

ASF is endemic in Africa where a sylvatic cycle between Ornithodoros spp. soft ticks and the natural reservoir, African wild suids, maintains its presence ${ }^{3}$. Other routes of infection and spread include physical contact, fluids, excretions, contaminated feed and fomites. The virus is extremely resilient and can survive for prolonged periods in a range of environmental conditions in carcasses and pork product. The disease is highly contagious and can be transmitted through relatively low infectious dose in feed and water ${ }^{4}$. Wild suid species are susceptible to disease but only domestic and feral pigs, as well as Eurasian wild boar show symptoms. ASFV is therefore very difficult to contain.

ASF was first discovered in East Africa, with symptoms reported in Kenya in 1914 and the disease described in $1921^{5}$. Outbreaks in other parts of Africa, Europe, Brazil and the Caribbean islands occurred in the $20^{\text {th }}$ century, with African countries being the worst affected. The virus was almost completely eradicated from non-African countries by the end of the century, but an outbreak in the Republic of Georgia in 2007 has since lead to widespread outbreaks in other countries. Since 2018 major outbreaks have been occurring in China, the world's largest pork producer, and it has been reported that up to half of the pigs in the country, representing roughly a quarter of the world's population, died or were culled to contain the outbreak in $2019^{6}$. The spread of the virus in East and Southeast Asia however could not be halted and since further countries including Mongolia, Vietnam, Cambodia, North Korea, Laos and island nations including The Philippines, Indonesia, 
Timor-Leste and Papua New Guinea have reported outbreaks ${ }^{7,8}$. After the initial outbreaks in Eastern Europe and continuing spread, the disease reached the European Union in 2014 and continues to spread, not least through the wild boar population ${ }^{7,9}$. In late 2020 the virus reached the largest producer of pork in Europe, Germany ${ }^{10}$. The virus is also edging closer to the USA, one of the world's main exporters and importers of pork, having been recently detected in Haiti and the Dominican Republic, only $381 \mathrm{~km}$ by air from the US territory of Puerto Rico. It is clear, that the disease represents a serious panzootic threat impacting the pork industry and threatening economies already shaken by the SARS-CoV-2 pandemic.

To understand the genetic and genomic variation for ASFV, sequencing is primarily focused on a roughly $400 \mathrm{bp}$ fragment of the $\mathrm{B} 646 \mathrm{~L}$ gene, encoding for the major capsid protein $\mathrm{p} 72$.

This fragment, representing $<0.25 \%$ of the total genome, is the basis of the current genotyping system, which has identified 24 genotypes so far ${ }^{11-14}$. To further discriminate, additional fragments of the E183L gene (p54, 630bp), CP205L (p30, 510bp), and B602L (gp83 $\sim 800 \mathrm{bp}$ ) are used, adding up to less than $1.4 \%$ of the genome characterized. Whilst being a DNA virus, antigenic diversity, the ability to acquire large deletions or insertions, and the presence of highly mutagenic hypervariable regions urge the need for whole genome sequencing for virus characterization and epidemiological studies ${ }^{15,16}$. To do this at the scale required, there is a need for a cheap and efficient method to sequence the large ASFV genome, whilst high abundance of homopolymers and hypervariable region require highest accuracy ${ }^{17}$.

The availability of a portable sequencing technology opens new doors to travel to outbreak locations, sequence, and analyze samples without needing to transport them. The MinION sequencer from Oxford Nanopore Technologies (ONT) can be carried easily in a pocket or carryon bag. This avoids complications of challenging transportation of biological samples, highly contagious agents or the requirement of a cold chain. There is the potential for a fast turnaround from sample collection to analysis, allowing for near live-monitoring of outbreak situations, as observed in during the Western African Ebola virus epidemic 2013-2016, or of 
bioRxiv preprint doi: https://doi.org/10.1101/2021.12.01.470769; this version posted December 3, 2021. The copyright holder for this preprint (which was not certified by peer review) is the author/funder, who has granted bioRxiv a license to display the preprint in perpetuity. It is made available under aCC-BY-NC-ND 4.0 International license.

course the COVID-19 pandemic. Furthermore, multiplexing and the washing and reuse of the most expensive component of sequencing, the flow cells, allowing for cheaper sequencing than other methods. Finally, the sequencer can produce very long reads which improves assembly potential, particularly of highly repetitive genomes.

Whilst it is possible to obtain whole genome sequences of ASFV directly from blood- and tissue extract DNA, the high prevalence of pig DNA and the need for baits or other methods to enrich ASFV DNA render that method inapplicable for high-throughput, fast, sequencing.

Here, we present a method to sequence the near complete genomes, excluding only the highly repetitive, variable length telomeric 3' and 5' regions, of ASFV using ONT's MinION sequencing device using a tiled amplicon approach. The genome is amplified in 32 large fragments $7 \mathrm{~kb}$ in length, amplified simultaneously in two PCR pools. We propose this method as an efficient, highly adaptable, more accurate, fast, and cost-effective option for sequencing of continuing ASFV outbreaks as well as historic samples. We present 10 complete ASFV genome assemblies from samples from the early stages of the ASFV outbreak in the Philippines in 2019 assembled either with the tiled sequencing approach or a whole genome sequencing shotgun approach. The portability of Nanopore sequencing makes it ideal for exploring the dynamics of ASFV infections as outbreaks emerge. As ASFV continues to spread around the world, efficient methods of sequencing the genome are essential to improve our understanding of the virus and the ongoing global spread. Our primer sets have been optimized for relatively even coverage and have been designed to bind outside of hypervariable regions. They only anneal to roughly $0.8 \%$ of the genome and are designed to be well suited to the current outbreak, able to at least partially sequence other genotypes and be easily modifiable should the virus mutate.

Finally, we present the Lilo pipeline. While pipelines exist to assemble genomes from tiled amplicons, they rely on aligning reads to a reference and using polishing tools to generate a consensus from the reads. This method works well for producing a genome sequence with SNPs representative of the sequenced genome, however large indels, structural variants, 
and hypervariable regions that may be difficult to align to a reference are not accurately represented. For ASFV, whole genes can be inserted or deleted and due to homologous recombination it can carry large structural variations, with indels likely being more important than SNPs in creating viral diversity ${ }^{18}$. Therefore, we designed Lilo, which aligns reads to a reference in order to assign them to an amplicon, selects the read with the highest base quality and of the expected length for each amplicon, polishes the read with the remaining reads, removes primers and stitches them together at overlaps ordered and oriented by a reference. This approach makes the pipeline more adaptable to large structural variation and hypervariable regions in genomes than currently available methods.

\section{Shotgun sequencing of ASFV directly from blood}

In field sequencing, particularly in developing countries, limits the availability of tools and reagents. During the first outbreaks in the Philippines whole DNA was isolated from the highly hemolysed blood collected from ASFV positive pigs. Samples were digested overnight with proteinase $\mathrm{K}$ at $55^{\circ} \mathrm{C}$ prior to phenol/chloroform/isoamyl alcohol extraction and precipitation with isopropanol before washing with $70 \%$ ethanol. Whole DNA samples were prepared for sequencing using the ligation sequencing kit (LSK) LSK109 before sequencing samples on a R9.4 flow cell using a MinION mk1b. The data were basecalled and demultiplexed using Guppy (ONT) and the reads assembled with Flye and polished with medaka. (Figure 1A)

The time between the beginning of sequencing and detection of the first ASFV read from whole blood ranged from 19 seconds to 3 minutes. As seen in the example of PHL-1969 (Figure 1B) the percentage of reads that came from ASFV ranged from $0.006 \%$ to $0.24 \%$, likely dependent on the viral titers of the animals culled. ASFV samples show a similar size distribution to other DNA found in the samples, if anything a second small peak of larger fragments can be observed (Figure 1C). All four sequenced blood samples assembled into a 
149 whole genome, however, due to variable coverage, the number of mismatches and indels

150 found in some of the samples were high (Figure 3B).

\section{Tiled amplicon sequencing of ASFV}

153 Given the low yield of ASFV sequences from shotgun sequencing, as demonstrated by us and others ${ }^{19-21}$, and the high expense per sample, this sequencing approach was not fit for purpose for high-throughput screening of an ongoing virus outbreak. Therefore, we developed a method to amplify, sequence, and assemble ASFV genomes from pigs.

In order to enrich ASFV from the sample easily, a PCR amplification approach was chosen, due to its ease of use and usually readily available tools in many countries and labs. Tiling primers were designed targeting $7 \mathrm{~kb}$ amplicon length and $1 \mathrm{~kb}$ amplicon overlap using primal scheme using a set of 26 ASFV reference sequences (Figure 2A). The primers are well suited to genotype II, from the current outbreak, but also cover the majority of the genome for at least genotypes I and IV (Figure 2B). This relatively long amplicon size was chosen to reduce the number of primer pairs but also to span potential hypervariable regions. After initial individual performance tests, several primers were redesigned from the original set of primers produced by primal scheme, however the majority of them worked well from the beginning. Fragments were amplified using the PCRBio VeriFi Hot Start high fidelity polymerase according to the manufacture's instruction. Following redesign, all primers amplified their targets, however, they did so at different efficiencies leading to uneven coverage over the genome. To test this, evenly concentrated pools of primers (pool 1 and pool 2, Figure 2A and Figure 2C) were used to amplify blood DNA extract samples from

171 ASFV-infected pigs. Following initial amplifications, pools were split into three pools with

172 primer pair 1 , producing a shorter $4 \mathrm{~kb}$ fragment continuously outperforming the others in a 173 mixed reaction on its own, and primer concentrations in pool 1(Pair 1) and pool 2 were 174 gradually adjusted according to their performance. PCR products per sample were 
bioRxiv preprint doi: https://doi.org/10.1101/2021.12.01.470769; this version posted December 3, 2021. The copyright holder for this preprint (which was not certified by peer review) is the author/funder, who has granted bioRxiv a license to display the preprint in perpetuity. It is made available under aCC-BY-NC-ND 4.0 International license.

175 combined, libraries prepared using the LSK109 kit in an R9.4 flow cell. Figure 2D

176 demonstrates the improvement that can be gained by tweaking primer concentrations from

177 evenly represented primer pairs (purple) to optimized primer concentrations (green). These

178 optimizations improve performance for multiplexing of multiple samples on one flow cell.

179 Fresher samples amplify more cleanly, but older, degraded samples will still amplify

180 sufficiently. To show this, we highlight two samples; sample PHL-126, which has been

181 heavily used and degraded, and sample PHL-261, which has been used less frequently

182 (aliquot stored in freezer without frequent use) and is of better quality. As can be seen in the automated electrophoresis result of a tapestation (Figure 2E), PHL-126 shows poor amplification and relatively many amplicons $<7 \mathrm{~kb}$. Good amplification can be seen for the shorter amplicon pair 1 still. PHL-261 on the other hand shows continued good amplification of the desired $7 \mathrm{~kb}$ and $4 \mathrm{~kb}$ products of pool 1 (odd), pool 2 (even) and pair 1 , respectively. These samples were prepared with the LSK109 kit and multiplexed using native barcoding and run on a R9.4 flow cell with 3 other ASFV genomes having been pooled in representative quantities, the poorer amplification of PHL-126 had lower sequencing throughput than the better quality PHL-261, but was still assembled into a near-complete genome. Figure $2 \mathrm{~F}$ shows sequencing coverage of the same two samples and the proportion of total reads for each that was assigned to each of the 32 amplicons.

The post-amplification DNA Integrity Number (DIN) can be used to help predict multiplexability, as different quality samples will impact the needed throughput. Figure $2 \mathrm{G}$ shows the relationship between the post-amplification DIN and throughput $<3 \mathrm{~kb}$, and Figure $2 \mathrm{H}$ demonstrates the number of gaps for different throughputs of reads $>3 \mathrm{~kb}$. Samples PHL126 (orange) and PHL-261 (blue) have been highlighted in pale (super accuracy base calling $(\mathrm{SAC})$ ) and strong (high accuracy base calling (HAC)) colors, respectively. Figure $2 \mathrm{H}$ demonstrates the required throughput per sample and the gaps that can be expected in genomes produced with lower throughputs. Assuming a theoretical MinION throughput of 
202 (including PCR control), however we would recommend starting with fewer and assessing

203 achievable throughput for each sequencing location as there is variability in expected

204 throughput between users, flow cells, and geographical location. The integrity of the sample

205 will also impact the throughput with degraded samples leading to sequencing capacity being

206 taken up by shorter fragments instead of the required full length amplicons (Figure $2 \mathrm{G}$ ). For

207 very poor samples, more stringent size selection with AMPure XP beads prior to sequencing

208 may be necessary if samples are to be multiplexed. While it is impractical to run an

209 automated, high resolution electrophoresis, such as a tapestation, after every amplification,

210 users can test a typical sample type (e.g. from decomposing wild boar, from farm culled pigs,

211 with/without cold chain) to predict the likely multiplexability and clean-up steps of similar

212 samples.

\section{Lilo assembly of genomes from tiled amplicons}

215 Comparing ASFV genomes we found major variation of the genome often originating from

216 indels. Available assembly pipelines were struggling with such variation when it did not

217 correspond to the reference sequence. Therefore, we developed the Lilo pipeline (Figure 3A)

218 to assemble the tiled amplicons (https://github.com/amandawarr/Lilo). Whilst Lilo uses a

219 reference alignment to sort the amplicons, it polishes against the highest quality reads rather than a reference sequence. Using this pipeline, highly accurate genomes were obtained with mismatch accuracy approaching Q50 when using SAC (Figure 2I) and indel accuracy up to

222 Q40 when compared to a closely related publicly available ASFV genome assembly

223 (MN715134.1 $)^{21}$, which may still be quite divergent from these samples in truth.

224 QUAST ${ }^{22}$ (v5.0.2; quality assessment tool for genome assemblies) results demonstrate that 225 the increased coverage of the tiled amplicons produced a more accurate assembly than 226 shotgun sequencing of the virus using a whole flow cell sequencing directly from extracted

227 DNA. Shotgun sequencing however, was able to highlight some samples with longer 
telomeric regions, such as PHL-237, which is a clear advantage of long-read sequencing

technology and something that should be explored for more in-detail investigations into the role of the ASFV telomeric regions. Overall, SAC produced fewer mismatches and indels than HAC and should be the preferred method, however, the time for base calling is a tradeoff. Samples with high percentages of unassigned bases (N's) clearly correspond to DIN numbers (Figure 3B).

The assembled genomes had excellent agreement on genome structure with the same samples assembled from shotgun sequencing (Figures 3B \& 3C). The repetitive content shown at the edges of Figures $3 \mathrm{~B}$ and $3 \mathrm{C}$ are sequences from the telomeres, showing that despite the sequences being from tiled amplicons, they do cover the majority of the genome and part of the telomeres.

\section{Accuracy of Lilo and ARTIC assembled genomes}

241 We assessed the quality of Lilo assemblies against those produced with the ARTIC pipeline

242 (v1.2.1). A selection of the ASFV sequencing data were assembled using the ARTIC

243 pipeline, as well as using Lilo, both using the assembled shotgun sequence PHL-1969 as a

244 reference.

245 QUAST analysis shows lower numbers of mismatches against the closest reference

246 (MN715134.1) but higher indels. The percentage of unassigned bases is much higher for

$247 \mathrm{ARTIC}$ at around $2.4 \%$ whereas Lilo is at 0 or nearly $0 \%$.(Figure $3 \mathrm{~B}$ )

248 Comparing Lilo-assembled genomes and ARTIC-assembled genomes to a reference

249 (MN715134.1) a number of indels can be observed. Figure 4A shows a likely real indel in the

250 PHL ASFV samples which all assemblies agree on and which is well supported by the reads.

251 In contrast, Figure 4B shows the only indel unique to almost all of the assemblies produced

252 by the Lilo pipeline while being absent from all artic assemblies and occurs in a

253 homopolymer, Most reads appear to support the deletion assembled by Lilo, whether this is 
a real sequence or a result of poor accuracy of Nanopore sequencing of homopolymeric regions is a more difficult question. Figure $4 \mathrm{C}$ shows an extreme example of a very long homopolymeric region, ASFV has several of these and typically neither assembly method agrees on the length of the homopolymer, with the reads lending no strong support to either assembly. While errors from the Lilo pipeline tended to be randomly dispersed among homopolymers, ARTIC errors tended to be more systematic, appearing consistently across the assembled genomes. Frequently, homopolymers lead to the ARTIC pipeline replacing the base immediately before the homopolymer and the first base of the homopolymer with a pair of N's, as can be seen in Figure 4E and 4F. There were also occasions when reads did not support an indel, and there wasn't a clear cause of an indel in the reads or reference, as can be seen in Figures 4D and 4E. There are several of these types of indels throughout the assembly, where Lilo assemblies better agree with both the reads and the reference, likely contributing to the lower QUAST scores of ARTIC on homopolymers and the percentage of undefined bases.

\section{Phylogenomics}

270 A maximum likelihood phylogenomic tree was constructed incorporating the newly

271 assembled genomes from tiled data of R9.4 flow cells with HAC with publicly available whole 272 genome ASFV sequences using iqtree (v2.0.5; Figure 5A), and for B646L gene, encoding for 273 the major capsid protein p72, genotype II sequences specifically (Figure 5B).

274 As observed in Figure 5A, p72 genotypes do not correspond to the clustering. For example,

275 the E75 strain Spain 1975 isolate, an early genotype II, is grouping with genotype l's.

276 Unfortunately, the phylogeny contains many gaps and lacks both timely and geographic

277 resolution, showing that much more sampling is required. PHL samples clearly cluster within 278 the highly virulent, novel p72 genotype II cluster. 
279 Resolving the tree further, selecting only those clustering with the novel p72 genotype II

280 genomes two distinct clusters of PHL sequences can be observed. Whilst, due to the

281 similarity of the genomes, the orders of lower branches are of lower confidence than those of

282 higher branches, reanalysis still suggest two different introductions into the Philippines

283 (internal branch lengths may be found in supplementary documents S1 and S2). Whilst

284 indications are that one cluster is closer related to Asian isolates, the other one showing a

285 more likely Eastern European origin, the lack of sample numbers to fill branch gaps and

286 common ancestors makes conclusive interpretation impossible. 


\section{Discussion}

288

ASFV is a serious threat to the global pork industry and consequences of depopulation, reduced availability of pork, and increased prices of other animal protein affect local economies, especially in low- and middle income countries with high reliance on pig protein. Tracing the spread of the virus, understanding more about genome-pathology links, and consequently implementing targeted biosafety measures are paramount to combat the disease. As demonstrated in Figure 5, current genotyping methods based on partial sequencing of the B646L gene, encoding for the major capsid protein $\mathrm{p} 72$, do not correspond to whole-genome sequencing and are therefore inadequate to trace virus evolution.

Here, we have demonstrated an efficient method for sequencing the ASFV genome. Despite the use of tiled amplicons, part of the 3'- and 5' telomeric regions of the virus are included in the tiled amplicon assemblies, meaning the majority of the genome is included.

As demonstrated by us and others, ASFV sequences can be obtained by direct sequencing from blood or other tissue samples of infected pigs ${ }^{19-21}$. The resulting sequence includes interesting information on the lengths and repeats found in the telomeric regions, which may be helpful for more in-depth investigation into the virus pathology and spread. However, without enrichment for ASFV ${ }^{17}$ or depletion of host-methylated DNA ${ }^{23}$ sample percentage for ASFV is low relative to host DNA in the samples, meaning that obtaining sufficient ASFV reads to assemble the genome from shotgun sequencing usually requires an entire MinION flow cell, or more, depending on viral titer and original sample type. Bone marrow or blood will likely yield the best virus:host ratio with spleen or muscle, whilst good sources of viral DNA ${ }^{24}$, also contain a large number of nucleated host cells. Even if sufficient data is obtained to assemble the genome, the coverage is likely too poor to sufficiently polish the genome. In contrast, the tiled amplicon method can be used on samples with lower viral titers or degraded DNA, selectively sequences the virus, and can be multiplexed on a flow cell to simultaneously sequence multiple samples at high enough coverage for good 
bioRxiv preprint doi: https://doi.org/10.1101/2021.12.01.470769; this version posted December 3, 2021. The copyright holder for this preprint (which was not certified by peer review) is the author/funder, who has granted bioRxiv a license to display the preprint in perpetuity. It is made available under aCC-BY-NC-ND 4.0 International license.

314 polishing. Especially in countries where ASFV is circulating in wild boar or feral pigs,

315 samples may be collected from infected animals that have been dead for a prolonged period 316 of time. It is important that the method is capable of amplifying virus from both high- and low 317 quality samples. Figure $2 \mathrm{E}$ demonstrates the variability of DNA integrity post-amplification 318 and that even poor samples that have been degraded amplify and produce near complete 319 genome assemblies.

320 Overall, the PCR amplification method increases coverage, is less prone to exhaust flow cells quickly, allows for multiplexing, and consequently reduces costs, improves genome accuracy, and removes the need for specialized enrichment or depletion methods.

Whilst $\sim 7 \mathrm{~kb}$ amplicons are very large compared to other comparable methods for other viruses, the size of the ASFV genome, the stability of DNA, the relatively low numbers of primer pairs, and the advantages of long reads detecting recombinants more easily make this the best approach. Especially with the small, medium, and large indels that can occur in ASFV ${ }^{18}$, it is important to get good resolution across these regions, which can be achieved easily with large amplicons. It is important though to choose the right, high accuracy polymerase capable of amplifying such long amplicons. We found PCRBio VeriFi to be highly capable of this with the hot start version producing very few non-specific products, whilst the non-hot start version can produce more non-specific product, which may be an advantage for variant testing. As demonstrated in Figures $2 E$ and $F$ and $4 B$ show that even low quality samples can produce whole genome assemblies with few gaps. However, a limitation of the large tiled amplicon method is that should a variant occur at the site of a primer, the amplification of a relatively large section of genome will fail. While this is an inconvenience, it will be simple to redesign a primer to replace the failed one or to act as an alternate primer. It is also possible to amplify across a larger region using the existing primers either side of the failed one, generating a $14 \mathrm{~kb}$ product, to sequence a larger region and design a primer from the sequenced amplicon. This was found to be possible using the VeriFi HS polymerase and allows for the method to adapt as the virus changes. 
341 Whilst Nanopore sequencing methods provide a lot of advantages, such as sequencing on

342 site, portability, and accessibility to less specialist communities, there are, as for any

343 sequencing method, drawbacks.

344 As demonstrated in Figure 1I \& 1J the choice of flow cell and basecalling method has an

345 impact on the accuracy of SNPs and indels. Generally, throughput is lower and required

346 input DNA is higher for R10.3 flow cells, however HAC on R10.3 is of comparable accuracy

347 to SAC on R9.4, with super accuracy on R10.3 being of even higher quality. SAC is very

348 slow and resource hungry, and when speed is important such as in an active outbreak,

349 R10.3 with HAC may be a good compromise for maximising efficiency with minimal sacrifice

350 of accuracy. However where the highest accuracy is required we recommend using R10.3

351 flow cells and basecalling with a super accuracy model.

352 All of our assemblies have indels compared to the reference partially stemming from

353 systematic errors in Nanopore sequencing in the abundant homopolymers and repeats in the ASFV genome (e.g. PHL-1969 contains $6.8 \%$ homopolymers of $4 \mathrm{bp}$ or more), however with the latest kits and flow cells from Nanopore, and without additional costs, we would expect future developments to continually reduce the indel errors without major alterations to the wet or dry lab methodologies described here. Additionally, some indels are likely true variations, and in many cases these indels are clearly present across all of our genomes (Figure 4A), and are not in homopolymeric regions, suggesting true variation between samples and the reference used. Additional sequencing of the same amplicons with a higher accuracy technology, such as Illumina could be used to polish the assemblies, however this would add time and cost. Reducing the number of false indels, where possible, is important, as the ASFV genome is known to have functionally relevant indels ${ }^{25}$. Polishing with an accurate reference can produce assemblies that are very accurate, however, these methods do not handle structural variants and hypervariable regions well. While the genomes sequenced here do not have any major indels compared to the reference used, diversity in 
samples is likely to reveal more of them. While errors from the Lilo pipeline tended to be randomly dispersed among homopolymers, ARTIC errors tended to be more systematic, appearing consistently across the assembled genomes. Errors occurring in the same position between genomes may be more likely to impact phylogenomic analysis than relatively random errors. The only consistent indel error found across the majority of the Lilo assembled genomes that was always absent in the artic genomes is shown in Figure 4B.

374 This region contains a homopolymers, which is typically difficult to correct from Nanopore 375 sequencing data, however while the ARTIC assembly more closely agrees with the 376 reference, the reads are well-supporting of the deletion found in the Lilo assemblies. It is not 377 unusual when carrying out multi-sequence alignments between whole ASFV genome sequences, even those constructed from reads from a higher accuracy sequencing technology, to find large homopolymers of variable length and it is unclear to what degree these are limitations of sequencing technologies as opposed to real variation.

The Lilo pipeline also has some limitations, it currently assumes that any structural variants will not change the length of any given amplicon by more than $5 \%$, it assumes that structural variants will not be dramatic enough to prevent alignment to the reference for the purposes of assigning reads to amplicons and ordering and orienting the polished amplicons. Lilo also assumes the reads will be the full length of the amplicon, making it incompatible with ONT rapid kits that utilize transposases. However, the strength of not relying on polishing reads aligned to a reference is beneficial for genomes where structural variation is expected to be important, and for species with hypervariable regions which may not align and polish well with a reference. The pipeline has been tested on tiled sequences from ASFV, Porcine

390 Reproductive and Respiratory Syndrome-1 \& -2, and SARS-CoV-2 (data not shown here) and can handle custom schemes for other viruses.

392 ASFV is very under-sequenced with only a small number of whole genome sequences 393 available and there is a need for an affordable way to sequence the virus at scale, as previously discussed ${ }^{17}$. While the majority of sequences produced from sequencing 
individual genes in the virus have been frustratingly similar ${ }^{26}$ reducing their usefulness in epidemiological studies, variants and large deletions have been observed across the genome, and these have been found to affect phenotypes ${ }^{25}$. As outbreaks continue to spread around the world and the amount of virus in circulation increases, these variations will likely increase in frequency. Additionally, there are few of the ancestral viruses from Africa sequenced, and these should be sequenced to understand the evolution of ASFV, particularly the loss of its dependence on the sylvatic cycle. Given the slow mutational rate of the virus, sequencing individual genes is unlikely to be informative and so to have a chance of seeing variants in the virus the whole genome must be sequenced. The ability to amplify the genotypes with our current scheme decreases with distance from genotype II, and additional primers will need designing in the future to improve coverage over other genotypes, however current coverage using this primer scheme is still likely to be of more use than the p72 gene alone. Coverage gaps can be resolved relatively easily as larger amplicons can be generated with flanking primers. Should primers on older or emerging samples fail, the altered region can be amplified using primers from either side of the failed amplicon, spanning the region, and the sequenced amplicon can be used to design new

411 primers for the region.

412 Phylogenetic trees of currently available whole-genome ASFV sequences highlight the 413 inadequacy of the p72 genotyping in reflecting similarities of ASFV on a genomic level. The 414 sparsity of whole-genome sequences hampers the ability to trace virus movement and 415 results in high levels of uncertainty in phylogenetic analyses. Running the maximum 416 likelihood tree analysis including the whole-genome sequences obtained from tiled 417 amplification in this manuscript reliably grouped samples from the early Philippines outbreak 418 of ASFV in November 2019 into two clusters. This indicates at least two potential 419 introductions of ASFV into the Philippines. Due to the lack of samples and resolution in the phylogenetic tree, no conclusion about countries or region of origins is possible. 
bioRxiv preprint doi: https://doi.org/10.1101/2021.12.01.470769; this version posted December 3, 2021. The copyright holder for this preprint (which was not certified by peer review) is the author/funder, who has granted bioRxiv a license to display the preprint in perpetuity. It is made available under ACC-BY-NC-ND 4.0 International license.

421 We have presented an efficient, low cost method for sequencing and assembling ASFV

422 which can be carried out in the lab or in the field during outbreaks. The Lilo pipeline is a

423 lightweight pipeline that can be run on a standard laptop with 16GB RAM and no internet

424 connection, making it ideal for in field bioinformatic analysis of ASFV and other viruses. 


\section{Methods}

\section{Samples}

427 Blood samples from outbreaks in central Luzon (Philippines) were collected following 428 depopulation of pigs within a defined containment radius. Blood samples were tested for 429 ASFV by PCR. Blood samples from ASFV-positive pigs were pooled at equal amounts by 430 farm before further processing.

\section{DNA extraction}

432 Blood samples were spun for $20 \mathrm{~min}$ at 3,000 rcf before decanting the supernatant. 5xTEN 433 buffer(0.05M EDTA, $0.5 \mathrm{M} \mathrm{NaCl}, 20 \mathrm{mg} / \mathrm{ml}$ Proteinase $\mathrm{K}, 20 \% \mathrm{SDS}$, in $0.05 \mathrm{M}$ Trix- $\mathrm{HCl}$, $434 \mathrm{pH} 8.0$ ) were added to a $1 \mathrm{x}$ final concentration before incubation overnight at $55^{\circ} \mathrm{C}$ in a 435 shaking water bath. Equal volumes of phenol were added and gently mixed. Following $20 \mathrm{~min}$ centrifugation at 3,000rcf the aqueous phase was transferred to a fresh tube. If the phase was very viscous, the phenol phase was re-extracted to improve yields. An equal volume of phenol/chloroform/isoamyl alcohol (25:24:1) was added to the aqueous phase before mixing and separation by centrifugation, $10 \mathrm{~min}, 3,000 \mathrm{rcf}$. The aqueous phase was transferred to a

440 fresh tube before addition of 1:10 3M sodium acetate and an equal amount of isopropanol.

441 Following $1 \mathrm{~h}$ incubation at $-20^{\circ} \mathrm{C}$, samples were spun for $10 \mathrm{~min}$ at $16,000 \mathrm{rcf}$ before washing 442 the pellet with $70 \%$ Ethanol. The pellet was dried and resuspended in nuclease-free water.

\section{Nanopore sequencing directly from DNA extracted from blood in the Philippines}

444 Samples were sequenced following Nanopore's SQK-LSK109 protocol on R9.4 flow cells on 445 a MinION mk1b. The protocol was started with 1ug of DNA as measured on an Implen

446 NanoPhotometer P330. The protocol was carried out as recommended by ONT with the 447 following modifications: The $20^{\circ} \mathrm{C}$ and $60^{\circ} \mathrm{C}$ incubations after the addition of NEB's FFPE repair and End-prep reagents were done for 30 minutes at each temperature instead of 5

449 minutes, and the room temperature incubation for the ligation reaction was done for 20 450 minutes instead of 10. During sequencing, two USB desk fans were pointed at the MinION to 
451 assist with maintaining appropriate temperature for the run in the above average "room

452 temperature" in the lab in the Philippines.

\section{Designing primers for tiled amplification}

454 Tiled primers were designed using Primal Scheme (v1.3.2) ${ }^{27}$. A set of 28 complete African

455 Swine Fever genomes (listed in Supplementary Document S3) were downloaded from NCBI

456 for primer design, which at the time were all that were available. Additionally three Filipino

457 whole ASFV genomes we had assembled from shotgun sequencing data were included. A

458 multi sequence alignment was carried out with Clustal Omega (in MEGA v7.0.2) ${ }^{28}$. Primal

459 Scheme was run to produce $7 \mathrm{~kb}$ amplicons with $1 \mathrm{~kb}$ overlap resulting in 32 overlapping

460 primer pairs in two non-overlapping pools. Primers were tested on samples and while the

461 majority worked first time, several had to be redesigned due to failed amplification or

462 preferential amplification of off-target regions. Redesigns were done using Primer-BLAST ${ }^{29}$,

463 targeting a similar region of the genome to the failed amplicon. Some primers amplified more

464 efficiently than others and in order to make the coverage of these as even as possible, some

465 primers were tweaked to have a different concentration. One primer pair (amplicon 1) was

466 shorter than the others in order to avoid highly repetitive sequence in the telomeres and it is

467 recommended to amplify it in a separate reaction to pools 1 and 2 to avoid

468 overrepresentation.

469 Amplification, library prep and sequencing of tiled amplicons

470 Tiled primers were initially tested individually at 200nM concentration using approximately

471 90ng ASF DNA and Phusion High-Fidelity PCR Master Mix with HF Buffer with 1mM added

$472 \mathrm{MgCl}_{2}$ (both New England Biolabs, Ipswich, MA, USA). Individual PCRs, in a $25 \mu$ l reaction

473 volume, underwent initial denaturation of 2 minutes at $98^{\circ} \mathrm{C}$, followed by 33 cycles of 10

474 seconds at $98^{\circ} \mathrm{C}, 30$ seconds annealing at $63^{\circ} \mathrm{C}$, and 4 minutes and 40 seconds extension

475 at $72^{\circ} \mathrm{C}$, followed by a final extension for 10 minutes at $72^{\circ} \mathrm{C}$. PCR products were then

476 examined using Tapestation Genomic DNA analysis (Agilent, Santa Clara, CA, USA). While 
477 a small amount of off-target amplification was tolerated, primers which produced strong off-

478 target bands or weak bands of the correct $7 \mathrm{~kb}$ size were redesigned.

479 Once the complete set of primers had been successfully designed to cover the complete

480 genome, the primers were pooled in equal amounts into two pools of non-overlapping

481 primers. These pools were tested using the same conditions as the individual PCRs, but in a $48250 \mu$ reaction volume and using $1 \mu \mathrm{M}$ of the primer pool. The resulting PCR products were 483 cleaned using $0.4 \times$ volume AMPure XP beads (Beckman Coulter, Indianapolis, IN, USA) to remove products smaller than approximately $2 \mathrm{~kb}$ in length, then pooled equally prior to sequencing. The cleaned PCR products were quantified using a Qubit ds DNA BR assay 486 (Invitrogen, Waltham, MA, USA) and combined in equimolar amounts to a total of $700 \mathrm{ng}$ for 487 library preparation according to the Native barcoding genomic DNA (with EXP-NBD104, EXP-NBD114, and SQK-LSK109)-Nanopore protocol.

Following bioinformatic analysis of sequencing data, primers which were found to be over- or under-performing were either redesigned or their contribution to the pool was adjusted accordingly, and the new primer pool tested as above in an iterative fashion. Ultimately 2 non-overlapping pools and a separate reaction for primer pair 1 were used to obtain the most even coverage and were processed as above, and pooled proportionally to the number of amplicons in each pool prior to sequencing. Additionally the polymerase was swapped from Phusion to VeriFi (PCRBIO) in a 25ul reaction using 2ul DNA per reaction, which has markedly better performance on the amplicons with far less off-target amplification. The PCR conditions for this polymerase were an initial denaturation of 1 minute at $98^{\circ} \mathrm{C}$, followed by 40 cycles of 15 seconds at $98^{\circ} \mathrm{C}, 15$ seconds annealing at $60^{\circ} \mathrm{C}$, and 4 minutes and 40 seconds extension at $72^{\circ} \mathrm{C}$, followed by a final extension for 5 minutes at $72^{\circ} \mathrm{C}$. AMPure XP

500 bead cleanup after PCR is optional, but recommended in samples with low DIN. Primer 501 sequences, recommended primer concentrations and recommended pooling quantities are 502 described in supplementary table S1, and any updates to these will be released on Lilo's 503 github page. 
504 Samples were sequenced following Nanopore's SQK-LSK109 or SQK-LSK110 protocol on

505 R9.4 or R10.3 flow cells (which combination is specified alongside relevant results) on a

506 MinlON mk1b or mk1c. The protocol was started with 1ug of pooled amplicons as measured

507 on a qubit using broad range reagents. For samples using multiplexing, the native barcoding

508 expansion kit from Nanopore was used following Nanopore's instructions when using SQK-

509 LSK109. For using the barcodes with SQK-LSK110, the instructions for SQK-109 were

510 followed until after the barcodes had been ligated on, at which stage the end prep was

511 repeated and we follow the standard protocol for library prep with SQK-LSK110 from after

512 the end prep step.

513 Bioinformatic processing of ASFV genomes sequenced with shotgun sequencing

514 The data were basecalled and demultiplexed using MinKNOW (v19.06.8; ONT) using "fast"

515 basecalling. Following basecalling the reads were aligned to an ASFV genome using

516 minimap2 to identify ASFV reads, the fast5s for these reads were extracted using

517 fast5_subset from the ont_fast5_api (https://github.com/nanoporetech/ont fast5 api) and

518 these were basecalled again using high accuracy basecalling. This was done to reduce

519 basecalling time, as this work was done locally in the field on a laptop without a GPU. The

520 reads were assembled with Flye (v2.6) ${ }^{30}$ and polished 3 times with Medaka (v0.7.1; ONT).

521 Comparisons of quantity of data produced and the proportion of which were ASFV reads

522 were done using NanoComp (v1.28.1) ${ }^{31}$.

523 Bioinformatic processing of ASFV genomes from tiled amplicons with Lilo

524 The data were basecalled and demultiplexed using Guppy (v5.0.14; ONT) using high or

525 super accuracy model on a GPU. The snakemake pipeline, Lilo

526 (https://github.com/amandawarr/Lilo), was developed and as summarised in Figure 3A,

527 takes the following steps:

528 1. Use Porechop (v0.2.3) to remove any sequencing adapters or barcodes that have $529 \quad$ made it through demultiplexing. 
2. Align to a reference with minimap2 (v2.22) ${ }^{32}$ and samtools (v1.12) ${ }^{33}$ and separate reads into amplicons by alignment position with bedtools (v2.30.0). ${ }^{34}$

3. Select reads of the expected amplicon length (+/-5\%) and subset to $300 \mathrm{X}$

4. Select the read with highest average base quality within $+/-1 \%$ of the median length of reads for the amplicon to be the "reference" (with bioawk v1); https://github.com/lh3/bioawk), remove any amplicons with fewer than 40 reads. Targeting the median length allows for flexibility for large insertions or deletions.

5. Pool amplicon reads and references back into their original non-overlapping pools.

6. Polish the pools $3 x$ with medaka (v1.4.4; ONT) and combine resulting polished amplicons.

7. Align to the reference with minimap2 and remove soft clipped bases (these likely represent missed barcodes or adapters) primers from the amplicons.

9. Merge the amplicons with scaffold_builder (v2.3) ${ }^{35}$.

545 The required input to Lilo are demultiplexed reads in fastq format in a directory named

546 "raw/", a reference fasta, a bed file of primer alignments (as output by primal scheme), and a

547 csv of primer sequences (if there are ambiguous bases it is advised to expand them first)

548 and a config file, described on the github page. It is adaptable to any species (with a single 549 genome fragment/chromosome) with any tiled primer scheme. The pipeline outputs a fasta

550 file containing the assembled genome.

\section{$551 \quad$ ARTIC assemblies}

552 A subset of genomes were also assembled using the Artic pipeline

553 (https://artic.network/ncov-2019; v1.2.1) following the bioinformatics SOP using the medaka

554 method.

\section{Quality control of assembled genomes}


556 Quast (v5.0.2) was used to compare the assembled genomes to the most closely related

557 publicly available ASFV assembly according to BLAST alignment (MN715134.1) ${ }^{21}$. Samples

558 where both WGS and tiled sequencing were used were compared for overall structure using

559 nucmer (v4.0.0beta2) ${ }^{36}$.

\section{Phylogeny}

561 The phylogeny analysis was limited to the tiled genomes, as these were the most accurate

562 assemblies, and publicly available genomes. These were aligned using Mafft $(\mathrm{v} 7.467)^{37}$ and

563 maximum likelihood trees constructed using iqtree $(\mathrm{v} 2.0 .5)^{38}$. 


\section{Acknowledgments}

566 We would like to thank the Bureau of Animal Industry and Milagros Mananggit for providing

567 us access to the valuable ASFV blood samples. We would also like to thank Central Luzon

568 State University and Clarissa Yvonne Domingo and Virginia Venturina and their families for

569 their amazing hospitality during all our visits to the Philippines.

570 We acknowledge financial support from the BBSRC Institute Strategic Programme grant

571 funding to The Roslin Institute (BBS/E/D/20241866, BBS/E/D/20002172, and

572 BBS/E/D/20002174) and BBSRC / Newton Fund Swine and Poultry research initiative grant

$573(\mathrm{BB} / \mathrm{R} 013187 / 1)$

\section{Author Contributions}

575 Outlined the study AW, NC, IV, CYJD, CTB; collected and provided samples RGM, MRM,

576 CYJD; performed experiments AW, NC, CN, IV, RP, LCC, TGB, and CTB; analyzed data

577 AW, NC, SL, and CTB; interpreted data AW, NC, CN, SL, VMV, CYJD and CTB; wrote

578 manuscript, AW and CTB, with contribution of other authors.

\section{Competing Interests Statement}

580 The authors declare no competing interests. 


\section{References}

$5821 \quad$ Blome, S., Franzke, K. \& Beer, M. African swine fever - A review of current knowledge. Virus Research 287, 198099, doi:10.1016/j.virusres.2020.198099 (2020).

585

2 Sánchez-Vizcaíno, J. M., Mur, L., Gomez-Villamandos, J. C. \& Carrasco, L. An

586

587 Update on the Epidemiology and Pathology of African Swine Fever. Journal of Comparative Pathology 152, 9-21, doi:10.1016/j.jcpa.2014.09.003 (2015).

588 Jori, F. et al. Review of the sylvatic cycle of African swine fever in sub-Saharan Africa

589

590

591

592

593

594

595

596

597

598

599 and the Indian ocean. Virus Res 173, 212-227, doi:10.1016/j.virusres.2012.10.005 (2013).

4 Niederwerder, M. C. et al. Infectious Dose of African Swine Fever Virus When Consumed Naturally in Liquid or Feed. Emerg Infect Dis 25, 891-897, doi:10.3201/eid2505.181495 (2019).

5 Eustace Montgomery, R. On A Form of Swine Fever Occurring in British East Africa (Kenya Colony). Journal of Comparative Pathology and Therapeutics 34, 159-191, doi:https://doi.org/10.1016/S0368-1742(21)80031-4 (1921).

6 Tian, X. \& von Cramon-Taubadel, S. Economic consequences of African swine fever. Nature Food 1, 196-197, doi:10.1038/s43016-020-0061-6 (2020).

7 Penrith, M. L. Current status of African swine fever. CABI Agriculture and Bioscience 1, 11, doi:10.1186/s43170-020-00011-w (2020).

600

601

602

603

604

605

606

607

608

609

610

611

612

8 Mighell, E. \& Ward, M. P. African Swine Fever spread across Asia, 2018-2019. Transbound Emerg Dis 68, 2722-2732, doi:10.1111/tbed.14039 (2021).

9 Gaudreault, N. N., Madden, D. W., Wilson, W. C., Trujillo, J. D. \& Richt, J. A. African Swine Fever Virus: An Emerging DNA Arbovirus. Front Vet Sci 7, 215, doi:10.3389/fvets.2020.00215 (2020).

10 Sauter-Louis, C. et al. Joining the club: First detection of African swine fever in wild boar in Germany. Transbound Emerg Dis 68, 1744-1752, doi:10.1111/tbed.13890 (2021).

11 Bastos, A. D. et al. Genotyping field strains of African swine fever virus by partial p72 gene characterisation. Arch Virol 148, 693-706, doi:10.1007/s00705-002-0946-8 (2003).

12 Quembo, C. J., Jori, F., Vosloo, W. \& Heath, L. Genetic characterization of African swine fever virus isolates from soft ticks at the wildlife/domestic interface in

613

614

615

616

617

618

619

620

621

622

623

624

625

626

627

628

629

630

631 Mozambique and identification of a novel genotype. Transbound Emerg Dis 65, 420431, doi:10.1111/tbed.12700 (2018).

13 Boshoff, C. I., Bastos, A. D., Gerber, L. J. \& Vosloo, W. Genetic characterisation of African swine fever viruses from outbreaks in southern Africa (1973-1999). Vet Microbiol 121, 45-55, doi:10.1016/j.vetmic.2006.11.007 (2007).

14 Achenbach, J. E. et al. Identification of a New Genotype of African Swine Fever Virus in Domestic Pigs from Ethiopia. Transbound Emerg Dis 64, 1393-1404, doi:10.1111/tbed.12511 (2017).

15 Malogolovkin, A. \& Kolbasov, D. Genetic and antigenic diversity of African swine fever virus. Virus Res 271, 197673, doi:10.1016/j.virusres.2019.197673 (2019).

16 Netherton, C. L., Connell, S., Benfield, C. T. O. \& Dixon, L. K. The Genetics of Life and Death: Virus-Host Interactions Underpinning Resistance to African Swine Fever, a Viral Hemorrhagic Disease. Front Genet 10, 402, doi:10.3389/fgene.2019.00402 (2019).

17 Forth, J. H., Forth, L. F., Blome, S., Höper, D. \& Beer, M. African swine fever wholegenome sequencing-Quantity wanted but quality needed. PLOS Pathogens 16, e1008779, doi:10.1371/journal.ppat.1008779 (2020).

18 Zhu, Z. et al. Homologous recombination shapes the genetic diversity of African swine fever viruses. Veterinary Microbiology 236, 108380,

632 doi:https://doi.org/10.1016/j.vetmic.2019.08.003 (2019). 
bioRxiv preprint doi: https://doi.org/10.1101/2021.12.01.470769; this version posted December 3, 2021. The copyright holder for this preprint (which was not certified by peer review) is the author/funder, who has granted bioRxiv a license to display the preprint in perpetuity. It is made available under aCC-BY-NC-ND 4.0 International license.

63319 Jia, L. et al. Nanopore sequencing of African swine fever virus. Sci China Life Sci 63,

634 160-164, doi:10.1007/s11427-019-9828-1 (2020).

635

20 O'Donnell, V. K. et al. Rapid Sequence-Based Characterization of African Swine

636

637

638

639

640

641

642

643

644

645

646

647

648

649

650

651

652

653

654

655

656

657

658

659

660

661

662

663

664

665

666

667

668

669

670

671

672

673

674

675

676

677

678

679

680

681

682

683

684

685

686

687 Fever Virus by Use of the Oxford Nanopore MinION Sequence Sensing Device and a Companion Analysis Software Tool. J Clin Microbiol 58, doi:10.1128/JCM.01104-19 (2019).

21 Olasz, F. et al. Short and Long-Read Sequencing Survey of the Dynamic Transcriptomes of African Swine Fever Virus and the Host Cells. Front Genet 11, 758, doi:10.3389/fgene.2020.00758 (2020).

22 Gurevich, A., Saveliev, V., Vyahhi, N. \& Tesler, G. QUAST: quality assessment tool for genome assemblies. Bioinformatics 29, 1072-1075, doi:10.1093/bioinformatics/btt086 (2013).

23 O'Donnell Vivian, K. et al. Rapid Sequence-Based Characterization of African Swine Fever Virus by Use of the Oxford Nanopore MinION Sequence Sensing Device and a Companion Analysis Software Tool. Journal of Clinical Microbiology 58, e0110401119, doi:10.1128/JCM.01104-19 (2021).

24 Fischer, M., Huhr, J., Blome, S., Conraths, F. J. \& Probst, C. Stability of African Swine Fever Virus in Carcasses of Domestic Pigs and Wild Boar Experimentally Infected with the ASFV "Estonia 2014" Isolate. Viruses 12, doi:10.3390/v12101118 (2020).

25 Gallardo, C. et al. Attenuated and non-haemadsorbing (non-HAD) genotype II African swine fever virus (ASFV) isolated in Europe, Latvia 2017. Transbound Emerg Dis 66, 1399-1404, doi:10.1111/tbed.13132 (2019).

26 Gallardo, C. et al. Genetic variation among African swine fever genotype II viruses, eastern and central Europe. Emerging infectious diseases 20, 1544-1547, doi:10.3201/eid2009.140554 (2014).

27 Quick, J. et al. Multiplex PCR method for MinION and Illumina sequencing of Zika and other virus genomes directly from clinical samples. Nature Protocols 12, 12611276, doi:10.1038/nprot.2017.066 (2017).

28 Sievers, F. et al. Fast, scalable generation of high-quality protein multiple sequence alignments using Clustal Omega. Mol Syst Biol 7, 539, doi:10.1038/msb.2011.75 (2011).

29 Ye, J. et al. Primer-BLAST: A tool to design target-specific primers for polymerase chain reaction. BMC Bioinformatics 13, 134, doi:10.1186/1471-2105-13-134 (2012).

30 Kolmogorov, M., Yuan, J., Lin, Y. \& Pevzner, P. A. Assembly of long, error-prone reads using repeat graphs. Nature Biotechnology 37, 540-546, doi:10.1038/s41587019-0072-8 (2019).

31 De Coster, W., D'Hert, S., Schultz, D. T., Cruts, M. \& Van Broeckhoven, C. NanoPack: visualizing and processing long-read sequencing data. Bioinformatics $\mathbf{3 4}$, 2666-2669, doi:10.1093/bioinformatics/bty149 (2018).

$32 \mathrm{Li}, \mathrm{H}$. Minimap2: pairwise alignment for nucleotide sequences. Bioinformatics 34, 3094-3100, doi:10.1093/bioinformatics/bty191 (2018).

$33 \mathrm{Li}, \mathrm{H}$. et al. The Sequence Alignment/Map format and SAMtools. Bioinformatics 25, 2078-2079, doi:10.1093/bioinformatics/btp352 (2009).

34 Quinlan, A. R. \& Hall, I. M. BEDTools: a flexible suite of utilities for comparing genomic features. Bioinformatics 26, 841-842, doi:10.1093/bioinformatics/btq033 (2010).

35 Silva, G. G. et al. Combining de novo and reference-guided assembly with scaffold_builder. Source Code Biol Med 8, 23-23, doi:10.1186/1751-0473-8-23 (2013).

36 Marçais, G. et al. MUMmer4: A fast and versatile genome alignment system. PLOS Computational Biology 14, e1005944, doi:10.1371/journal.pcbi.1005944 (2018).

37 Katoh, K., Misawa, K., Kuma, K. i. \& Miyata, T. MAFFT: a novel method for rapid multiple sequence alignment based on fast Fourier transform. Nucleic Acids Research 30, 3059-3066, doi:10.1093/nar/gkf436 (2002). 
bioRxiv preprint doi: https://doi.org/10.1101/2021.12.01.470769; this version posted December 3, 2021. The copyright holder for this preprint (which was not certified by peer review) is the author/funder, who has granted bioRxiv a license to display the preprint in perpetuity. It is made available under aCC-BY-NC-ND 4.0 International license.

Minh, B. Q. et al. IQ-TREE 2: New Models and Efficient Methods for Phylogenetic Inference in the Genomic Era. Molecular Biology and Evolution 37, 1530-1534, doi:10.1093/molbev/msaa015 (2020).

691 


\section{$692 \quad$ Figure Legends}

\section{Figure 1}

694 A) DNA was extracted from blood and sequenced with Nanopore's LSK109 on an m1kb before analysis and assembly using Flye and polishing with medaka. B) Read length histograms for the dataset demonstrating total throughput (blue) and ASFV reads throughput

697 (orange) C) Normalized counts by dataset of reads for total throughput (blue) and ASFV

698 reads throughput (orange). D) Throughput over time for total read count (blue) and ASFV 699 read count (orange).

$700 \quad$ Figure 2

701 A) Design of the tiled primer scheme for ASFV with $\sim 7 \mathrm{~kb}$ amplicons and $\sim 1 \mathrm{~kb}$ overlaps. B)

702 Predicted primer binding in correct region for representative ASFV genotypes. C) Workflow 703 with extraction from blood, PCR amplification of primer pools, pooling, sequencing and bioinformatic analysis D) Coverage of one sample (PHL-3142) amplified with either evenly represented primer pairs (purple) or optimized proportions of primer pairs (green). E)

706 Tapestation capillary electrophoresis of amplified pools of two different samples, the low 707 quality PHL-126 and the high quality PHL-261, and statistics of the resulting assemblies of each of these. F) Coverage of amplicons using optimized primer concentrations for the two samples from figure 1E. G) Impact of post-amplification DIN on proportion of reads $<3 \mathrm{~kb}$ in length, essentially wasted sequencing capacity, using R9.4 flow cells and LSK-109

711 (magenta) or R10.4 and LSK-110 (blue). Semilog fit analysis shows an R squared correlation of 0.6420 or 0.7244 , for R9.4_109 and R10.4_110, respectively. H) Association

713 between overall throughput $>3 \mathrm{~kb}$ and number of gaps in final assembly using HAC

714 (black/bold) and SAC (grey/faint). A log-log analysis shows an R squared correlation of

7150.9680 and 0.9358 for HAC and SAC, respectively. PHL-126 is highlighted in orange and

716 PHL-261 in blue. I) Assembly accuracy based on proportion of mismatches against

717 reference (MN715134.1), with lines showing Q40 and Q50 PHRED scores. J) Assembly 
718 accuracy based on proportion of indels against reference (MN715134.1), with lines showing

719 Q30 and Q40 PHRED scores. A\&C Created with BioRender.com

720 Figure 3

721 A) Directed acyclic graph showing the steps the Lilo pipeline takes during assembly. The

722 graph has been simplified to show assembly of a genome containing 2 amplicons

723 (amplicon_01 and amplicon_n) for a single sample. B) Quast results for genomes

724 sequenced with tiled amplicons or from shotgun sequencing on R9.4 flow cells using SQK-

725 LSK109. Note PHL-10 and PHL-30 were sequenced with an earlier version of the primer

726 scheme with one primer different and are expected to have a 25bp gap C) Nucmer

727 alignment of PHL-261 genomes assembled from WGS or Lilo tiled assembly. D) Nucmer

728 alignment of PHL-237 genomes assembled from WGS or Lilo tiled assembly.Figure 4

729 B) IGV image showing alignment of genomes assembled with Lilo or Artic (top) and

730 assemblies and reads for a single sample (PHL-10) aligned to a reference (MN715134.1).

731 This image shows a likely real indel present in all assemblies and supported by the reads. C)

732 as in B, but showing an indel common in Lilo assemblies and missing in Artic assemblies

733 and the reference. D) as in B, but showing a long homopolymer with poor consensus from

734 the reads and inconsistent results in assemblies. E, F \& G) Examples of indels specific to the

735 Artic pipeline assemblies which do not agree with the reference.

\section{$736 \quad$ Figure 5}

737 Maximum likelihood trees for our R9.4/SQK-LSK109 genomes and A) all available ASFV

738 genomes downloaded from NCBI (09/11/2021) or B) those specifically clustering with

739 genotype II. 

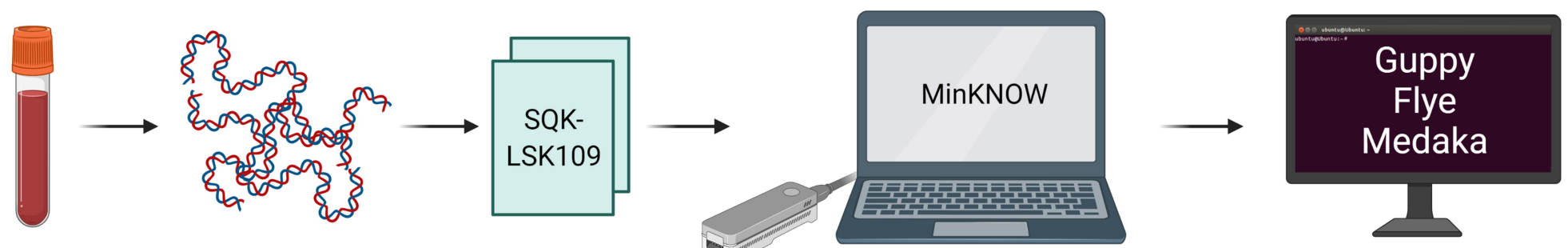

B

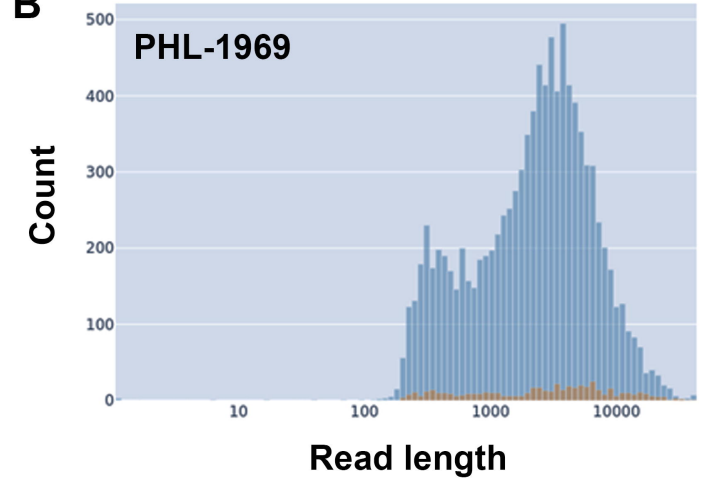

C

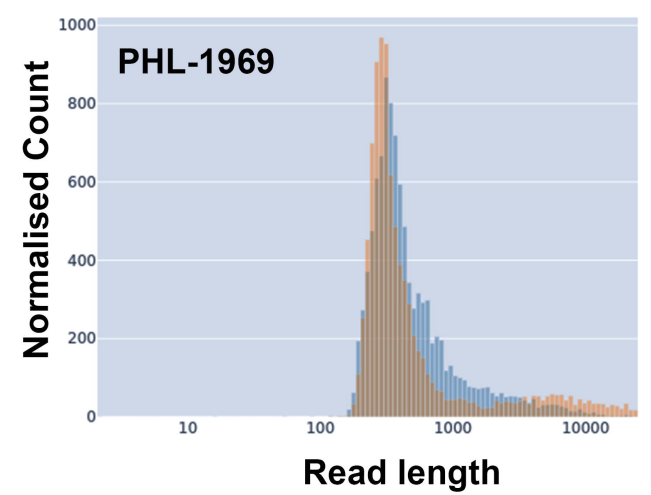

D

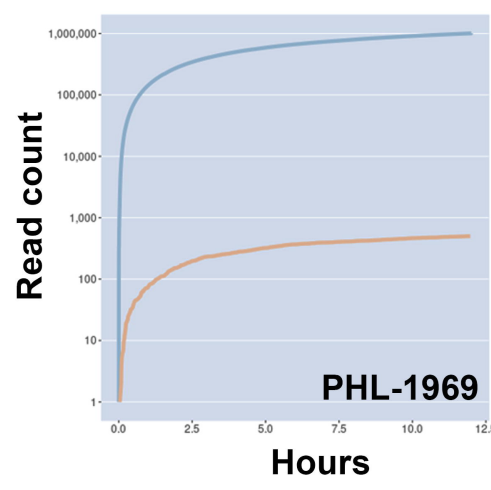


